\title{
Catherine Kintzler, Penser la laïcité
}

Minerve, 2014, 224 p.

Jean-Pierre Véran

\section{CpenEdition}

\section{Journals}

Édition électronique

URL : https://journals.openedition.org/ries/4479

DOI : $10.4000 /$ ries. 4479

ISSN : 2261-4265

Éditeur

France Education international

Édition imprimée

Date de publication : 1 décembre 2015

Pagination : 33-35

ISSN : $1254-4590$

Référence électronique

Jean-Pierre Véran, "Catherine Kintzler, Penser la laïcité », Revue internationale d'éducation de Sèvres [En ligne], 70 | décembre 2015, mis en ligne le 18 janvier 2015, consulté le 01 juillet 2021. URL : http:// journals.openedition.org/ries/4479; DOI : https://doi.org/10.4000/ries.4479

Ce document a été généré automatiquement le 1 juillet 2021.

(c) Tous droits réservés 


\section{Catherine Kintzler, Penser la laïcité}

Minerve, 2014, 224 p.

Jean-Pierre Véran

\section{RÉFÉRENCE}

Catherine Kintzler, Penser la laïcité, Minerve, 2014, 224 p.

1 S'il est une question vive partagée dans beaucoup de pays en 2015, c'est bien celle de la relation entre le politique et le religieux. De Singapour à la Tunisie, de l'Afrique du Sud à la Norvège, la question fait débat. En France, elle structure fortement le débat politique depuis le XVIII ${ }^{\mathrm{e}}$ siècle et a donné lieu à l'émergence, à l'affirmation constitutionnelle du principe de laïcité au XX $\mathrm{XX}^{\mathrm{e}}$ siècle. Aujourd'hui, le débat sur le lien ou la séparation entre le politique et le religieux, la loi et la foi suscite bien des prises de positions controversées.

2 Catherine Kintzler, professeur honoraire à l'Université de Lille, vice-présidente de la société française de philosophie, est notamment connue pour ses travaux sur Condorcet, l'instruction publique et la naissance du citoyen (Gallimard, 1987) et sur la laïcité (Qu'est-ce que la laïcité ?, Vrin, 2008). Le lecteur n'est donc pas surpris que la référence à Condorcet soit l'un des fils conducteurs de son approche rationnelle de la laïcité. Ses références philosophiques permettent de situer la réflexion de l'auteure : Descartes, Kant, Hegel et Bachelard sont parmi les plus cités. Il s'agit pour Catherine Kintzler de « rapatrier la laïcité dans la pensée des Lumières ", en commençant par expliciter le concept de laïcité.

3 Pour ce faire, elle s'efforce, dès le premier chapitre, en confrontant la pensée de Locke à celle de Condorcet, de distinguer le principe de toleration (tolérance) de celui de laïcité. La question est de savoir si le lien politique est inspiré par le modèle religieux (Locke), ou si l'on abandonne toute référence ou modélisation religieuse pour penser le lien politique (Condorcet). L'opposition de Condorcet à Robespierre à propos du culte officiel de l'Être suprême s'explique par cette distinction nette entre la loi et la foi. 
4 Le paradoxe de la laïcité tient à ce que le lien politique assure à chacun un maximum de liberté, en se fondant sur le droit de chacun de n'être pas comme le reste des hommes, pourvu qu'il respecte la loi.

5 Cette approche conceptuelle de la laïcité est indispensable pour apporter les réponses aux vraies et fausses questions que l'actualité française pose à propos de la laïcité, mais aussi pour comprendre la place particulière du régime de laïcité français par rapport aux accommodements raisonnables que permet la toleration dans d'autres États.

6 Catherine Kintzler pointe deux dérives françaises: celle de la laïcité adjectivée (plurielle, ouverte, etc.) et celle de l'intégrisme laïque. En particulier, elle met en garde contre les appels au «vivre ensemble », qui font l'impasse sur la nécessité d'assurer le vivre séparément pour vivre vraiment ensemble. La nation ne saurait être un agrégat de communautés, et l'école est le lieu fondamental de ce dépaysement social, où s'institue le citoyen. Combattant un courant de pensée qui voudrait que l'école de la République soit faite pour la République et qu'on y professe un catéchisme républicain fondé sur des valeurs inculquées, elle rappelle que l'école est faite par la République, mais pas pour la République. L'objectif de l'école est en effet d'établir des sujets de droits, des esprits autonomes, en respectant l'autonomie des savoirs. S'il fallait caractériser la laïcité d'un seul mot, ce serait sans doute l'a-dogmatisme qu'il faudrait choisir, ce qui est incompatible avec tout intégrisme prétendument laïc.

7 Connaître les religions n'est pas les reconnaître. À ce sujet, C. Kintzler montre le caractère doublement réducteur de ce que l'on appelle en France « l'enseignement du fait religieux ». Les religions, d'une part, ne sont pas qu'un fait, mais une pensée, une culture. Et, d'autre part, le fait religieux lui-même n'est pas incontournable. De même, elle exprime de vives réticences à l'égard d'un enseignement de la morale républicaine, qui constitue selon elle une impasse: c'est l'instruction qui produit la sérénité contemplative, non la morale qui évite la violence. Plaidant pour une école des humanités, elle distingue le fait de s'instruire en intériorisant la rationalité et celui d'apprendre, où la rationalité reste extérieure au sujet. L'objectif de tout enseignant, de toute leçon, est non pas que l'élève apprenne, mais qu'il s'instruise.

8 Son interprétation de la trilogue républicaine est éclairante : la fraternité est l'effet moral d'une liberté dont le moyen est l'égalité. Les citoyens sont à la fois rigoureusement identiques et absolument différents. Autrui n'est pas mon prochain, mais mon semblable. C'est en ce sens que les humanités intériorisées à l'école font de chacun un membre de l'humanité.

9 Le lecteur de cet ouvrage trouvera un grand plaisir intellectuel à penser la laïcité comme l'y invite l'auteure. La rigueur rationnelle du propos, la clarté des tableaux qui en résument la substance suscitent le plus grand intérêt. Les familiers du sujet et de Condorcet trouveront une preuve supplémentaire de cette rigueur intellectuelle devant la divergence affichée par $C$. Kintzler à l'égard de celui qui constitue pour elle une référence majeure, à propos de la conception d'un enseignement rigoureusement moderne: Condorcet, au siècle des Lumières, ne trouvait pas indispensable l'apprentissage du latin et du grec...

10 Ceux qui suivent le débat philosophique et politique sur la laïcité pourront mettre en tension utile la réflexion de Catherine Kintzler avec celle de Béatrice Mabilon-Bonfils et Geneviève Zoïa dans La laïcité au risque de l'Autre, publié la même année aux éditions de l'Aube. 
11 Dans les deux ouvrages, la réflexion s'empare des questions d'actualité, exprime la même réticence face à l'enseignement de la morale laïque, et la question de l'autre y est centrale. Mais si, comme on l'a vu, pour C. Kintzler, autrui n'est pas mon prochain mais mon semblable, G.Zoïa questionne cette approche philosophique au nom d'une approche sociohistorique et anthropologique. Et si l'universalisme citoyen de la République était le paravent d'une identité coloniale méconnaissant l'altérité des colonisés et de ceux qui en sont issus? Et B. Mabilon-Bonfils formule les plus vives critiques sur le résultat du programme d'instruction publique de l'école laïque, caractérisé par le renforcement des inégalités sociales et culturelles.

12 On retiendra donc de l'ouvrage de Catherine Kintzler sa contribution à -l'élucidation du principe et du régime de laïcité, contribution philosophique à un débat largement ouvert à d'autres approches complémentaires, voire contradictoires, sociohistoriques et anthropologiques. Les lecteurs pour qui les débats français semblent abscons y trouveront quelques utiles clés de compréhension, notamment de la distinction entre toleration et laïcité.

\section{AUTEURS}

\section{JEAN-PIERRE VÉRAN}

Jean-Pierre Véran est inspecteur d'académie honoraire. Il intervient en formation de l'encadrement sur la gouvernance des organisations éducatives, les politiques éducatives et l'éducation aux médias et à l'information. Blog : [http://blogs.mediapart.fr/blog/jean-pierreveran]. Courriel : jeanpierreveran@gmail.com 